\title{
Mathematical modeling of the microtubule dynamic instability: a new approch of GTP-tubulin hydrolysis
}

\author{
Ayuna Barlukova ${ }^{1}$, Stéphane Honoré ${ }^{2}$, and Florence Hubert ${ }^{1, a}$ \\ ${ }^{1}$ Aix-Marseille University, CNRS, Centrale Marseille, I2M UMR 7373, Marseille cedex 13, France \\ ${ }^{2}$ Aix-Marseille University, INSERM, CRO2, UMR_S 911, France
}

\begin{abstract}
Microtubules, components of the cytosqueleton, play an important role in cell division, cell migration and thus in the cancer proccess through dynamic instability. Therefore they are an important target for anti-cancer treatment. Proper modelling of dynamic instability is a crucial tool to understand the mechanism of action of microtubule targeting agents. In this paper, we propose a new concept for GTP-tubulin hydrolysis which allow the model to accurately reproduce microtubule dynamics observed in vitro or in cells. This approach will be more appropriate to take study the effects of drugs.
\end{abstract}

\section{Introduction}

MTs are cytosqueletal components found in all eukaryotic cells that play an important role in many cellular functions. They are long tubular polymers of $\alpha$ - and $\beta$-tubulin dimers. Segregating the chromosomes during mitosis, and regulating substrate adhesion dynamics and cell polarity during cell migration, MTs are involved in all crucial processes of cancer progression. MTs key property is their highly dynamic behavior. Indeed, they undergo transitions between sustained periods of elongation and rapid shortening. This phenomenon is referred in literature as MT dynamic instability. MTs have a polar structure : one of the ends has $\alpha$-tubulin exposed, "-end", while another end has $\beta$-tubulin exposed, "+end". Dynamic instability is observed either in vitro with pure tubulin at both end of the MT, suggesting that it is an intrinsic property of tubulin/microtubule, or in cells where it is regulated by several associated proteins both at the +end and the -end [AH15].

Although MTs can be dynamic at both ends in vitro, generally, only the +end dynamics is considered in modeling, since variations are much faster and larger for the +end than that for the -end. Moreover in vivo, MT -ends are most often anchored at the nucleation site (MT organizing center). A MT polymerizes by addition of free GTP-tubulin heterodimers to the +end until catastrophe, a switch toward the depolymerization state. Return of a depolymerizing MT into polymerization state is termed rescue. Transition from growing to shortening, i.e. the catastrophe event, is crucial for the dynamic instability and is thus more throughly studied. Biological observations show that microtubule body is mainly composed of GDP-tubulin and several layers of GTP-tubulin at the growing +end, form the "GTP-cap". The catastrophe is always accompanied by the loss of the GTP-cap. The GTP-cap has long been postulated to exist, but recent experiments give the first quantitative measurement in cells since the discovery of the ability of EB proteins to sense the nucleotide state of tubulin [MFB +12$]$.

\footnotetext{
a e-mail: florence.hubert@univ-amu.fr
} 
Indeed, during MT growth, GTP-tubulin is hydrolysed in GDP-tubulin shortly after incorporation. The existence of the GTP-cap is regulated by the balance between rate of polymerization and rate of hydrolysis. Moreover, a recent study suggests that catastrophe frequency depends on MT "age" ([GZG+11]). Indeed, young MT are protected from catastrophe while older ones are more prone to depolymerization. As there is more and more arguments for catastrophe is not a single-step process, there is a need of new simulation models that consider catastrophe as a multistep process. Several assumptions can be proposed to explain the multistep process of catastrophe. Here, we propose a new ansatz where this multistep process is link to GTP-hydrolysis. Indeed, observing that EB-GTP cap undergoes alterations during the course of MT growth, we propose that hydrolysis rate is an increasing step function of the age of MT, defined as the time after the MT growth or regrowth (rescue).

To better understand the complex process of MT dynamic instability, mathematical modeling has been used by several teams. Several strategies have been developped: modeling the dynamics of either a small number of MT (see eg [HRL+11] for a stocchastic approach), or of a MT-population ([HRT09]). These approaches are often complementary and more complex they are, more difficult is the calibration with data from biological observations: average length, catastrophe frequency, rescue frequency, growth rate, shortenning rate and so on. Aging effect has been taken into account in few models as in [GZG+11], [JG15] at macroscopical level.

Our aim is to take into account the impact of aging at the microscopical level and to evaluate the global consequence on the population of MT. We extend here a MT population model proposed by [HRT09]. The model expresses the evolution of concentrations of free GTP-, and GDP-tubulin, and densities of MTs that are in state of growth and shortening without any details on mechanical properties of a MT structure. The density of the population of growing MT in our new model in structured not only by time, length of the MT, length of the GTP-cap, but also by "age". This new variable "age" will allow us to take into account the age-dependance of the hydrolysis. Note that the mecanism of hydrolysis has not been fully elucidated, Through many computational and analytical models of MT dynamic instability, it emerges different ideas on mechanism of hydrolysis : random, vectorial or coupled random hydrolysis ([MK14], [HRT09], [HRL+11]). Since hydrolysis leads to catastrophe, choice of the mechanism of hydrolysis is one of the crucial points to simulate dynamic instability. We propose here a new concept for GTP-tubulin hydrolysis where hydrolysis is mainly vectorial, age-dependant and delayed according to the incorporation in the MT.

\section{Model and results}

The mathematical model of MT dynamic instability that we consider is based on the continuous approach of P. Hinow et al. [HRT09]. The originality of our model is that each MT is characterized by the elapsed time since the last instant of rescue or nucleation, that we call "age", to simulate the impact of aging properties on MT population. By aging we mean the phenomenon that older MTs are more prone to undergo catastrophe than younger MTs. Dynamics is generated by evolution of quantity of free GTP tubulin that is described by the function $t \rightarrow p(t)$, quantity of free GDP tubulin $t \rightarrow q(t)$ and by evolution of population of MTs. We distinguish MTs in depolymerization state with the density $v(t, \cdot)$ and in state of growth $u(t, \cdot, \cdot, \cdot)$. MTs in depolymerization state do not possess a GTP stabilizing cap so that this population can be described by one space variable $x$ corresponding to a length of a MT. A growing MT is characterized by three variables such as the total length $x$, the length of a GTP stabilizing cap $z$ and the variable age $a$. Age has a dimension of time and it is counted from zero after a MT undergoes rescue or nucleation. The equation for $u$ is a transport equation

$$
u_{t}+u_{a}+\gamma_{p o l}(p(t)) u_{x}+\left(\gamma_{p o l}(p(t))-\gamma_{h y d r o}(a)\right) u_{a}=0, x \in(0, \infty), z \in(0, x) t \in(0, \infty), a \in(0, \infty),
$$


where $\gamma_{p o l}(p)=\alpha_{p} *\left(p>p_{s}\right)+\left(\alpha_{p} \frac{p(t)-p_{c}}{p_{s}-p_{c}}\right) *\left(p_{c}<p<p_{s}\right)$ is a growth velocity of a MT +end and $\gamma_{\text {hydro }}(a)=\gamma_{h}^{\text {old }} *\left(a_{s}<a\right)+\gamma_{h}^{\text {young }} *\left(a_{\text {deco }}<a<a_{s}\right)$ is a velocity of the progression of the front between GDP and GTP dimers that we will refer here as rate or velocity of hydrolysis. The thresholds $p_{c}, p_{s}$ correspond to saturation and critical values of concentrations of free GTP tubulin, $\alpha_{p}$ stands for the maximal growth rate, $a_{\text {deco }}$ is the time necessary for one freshly incorporated dimer to be hydrolyzed, $a_{s}$ is an switching age for which hydrolysis rate raise up from $\gamma_{h}^{\text {young }}$ to $\gamma_{h}^{\text {old }}$.

The equation (1) is endowed with boundary conditions

$$
\gamma_{h y d r o}(a) u(t, a, x, x)=\mu p^{2} \psi(x) \Theta(a), R(t, a) u(t, a, x, 0)=\Theta(a) \lambda v(t, x) \text { if } R(t, a)>0,
$$

where $R(t, a)=\gamma_{p o l}(p(t))-\gamma_{\text {hydro }}(a), \mu$ is a nucleation rate, $\lambda$ characterizes the propensity of shrinking MTs to be rescued, $\Theta$ and $\psi$ are nonnegative functions such that $\int_{0}^{\infty} \Theta(a) d a=1, \int_{0}^{\infty} x \psi=1$ supported respectively on supp $\Theta=\left(0, a_{0}\right)$ and supp $\psi=\left(0, x_{0}\right)$. The equation for $v$ is also a transport equation

$$
v_{t}-\delta v_{x}=-\int_{0}^{\infty} R(t, a) u(t, a, x, 0) d a, x \in(0, \infty), t \in(0, \infty)
$$

where the parameter $\delta$ is the velocity of depolymerization of MTs. The last two variables $p$ and $q$ are solution of ODE :

$$
p_{t}=-\gamma_{p o l}(p) \int_{0}^{\infty} \int_{0}^{\infty} \int_{0}^{x} u(t, a, x, z) d z d x d a+k q-\mu p^{2}, q_{t}=\delta \int_{0}^{\infty} v(x, t) d x-k q, t \in(0, \infty) .
$$

The constant $k$ denotes a rate of recycling of GDP- into GTP-tubulin. The main property of this system is to preserve the total amount of tubulin

$$
L_{u}(t)+L_{v}(t)+p(t)+q(t)=C t e \text { where } L_{v}(t)=\int_{0}^{\infty} x v(t, x) d x, L_{u}(t)=\int_{0}^{\infty} \int_{0}^{\infty} \int_{0}^{x} x u(t, a, x, z) d z d x d a,
$$

Note that one of the output of model is the time evolution of temporal frequence of catastrophe:

$$
F_{\text {cata }}^{\text {temp }}(T)=\frac{1}{T} \int_{0}^{T}\left(\int_{0}^{\infty} \int_{0}^{t} \frac{1}{a}(R(t, a)<0) u(t, a, x, 0) d a d x\right)\left(\int_{0}^{\infty} \int_{0}^{t}(R(t, a)<0) u(t, a, x, 0) d a d x\right)^{-1} d t .
$$

A finite volume approach can be use to approximate solutions to the system (1)-(3) ([BHHH15]). An example of output of the system is given in Figure 1 for parameters given in Table 1.

\begin{tabular}{|l|l|l|l|l|l|l|l|l|l|l|l|}
\hline parameters & $p_{c}$ & $p_{s}$ & $\alpha_{p}$ & $a_{\text {deco }}$ & $a_{s}$ & $\gamma_{h}^{\text {young }}$ & $\gamma_{h}^{\text {old }}$ & $\delta$ & $\lambda$ & $\mu$ & $k$ \\
\hline values & 2 & 4 & 10 & 0.1 & 1 & 7.4 & 7.6 & 15 & 0.136 & $1.568 \mathrm{e}-18$ & 1.2 \\
\hline
\end{tabular}

Table 1. Example of biologically relevant parameters

\section{Discussion}

The parameters in Table 1 have been taken in [HRT09] for some of them, or estimated according to the microtubule growth rate at steady state such as $\gamma_{h}$ or estimated with biological observations $a_{d e c o}$. This 

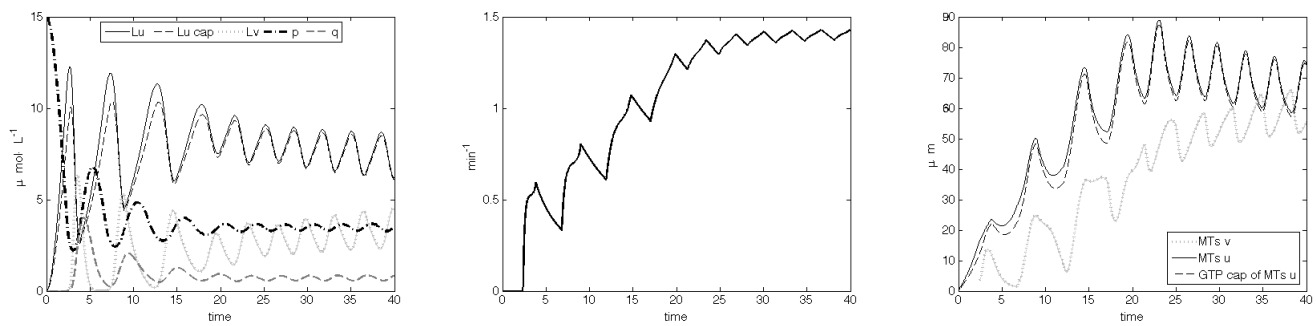

Figure 1. Time evolution of (Left) total concentration of the populations. (center) temporal frequence of Catastrophe. (Right) mean size of MT.

model enables us to reproduce key parameters of microtubule dynamic instability, and particularly catastrophe frequences observed either in vitro or in cells with values ranging from 1.0 to $2.5 \mathrm{~min}^{-1}$ as shown in Figure 1. Note that other indicators of microtubule dynamics are also in accordance with biological observations. Such model is also appropriate to study the impact of microtubule targeting agents, since all key parameters affected by the drug such as critical concentration $p_{c}, a_{d e c o}$. A confrontation with pharmacological data has to be performed in order to validate the model. In fine such model will allow us to better understand the way the drugs affect microtubule dynamics.

\section{References}

[AH15] A Akhmanova and CC Hoogenraad, Microtubule minus-end-targeting proteins, Curr Biol 16 (2015), no. 4, 25.

[BHHH15] A Barlukova, G. Henry, S. Honoré and F. Hubert, Dynamic instability of microtubules with aging and impact of drugs Submitted, 2015.

[GZG+11] MK Gardner, M Zanic, C Gell, V Bormuth, and J Howard, Depolymerizing kinesins kip3 and mcak shape cellular microtubule architecture by differential control of catastrophe, Cell 147 (2011), no. 5, 1092-103.

[HPB05] S Honore, E Pasquier, and D Braguer, Understanding microtubule dynamics for improved cancer therapy, Cell Mol Life Sci 62(24) (2005), 3039-56.

[HRL+11] P Hinow, V Rezania, M Lopus, MA Jordan, and JA Tuszyński, Modeling the effects of drug binding on the dynamic instability of microtubules, Phys Biol 8 (2011), no. 5, 056004.

[HRT09] P Hinow, V Rezania, and JA Tuszyński, Continuous model for microtubule dynamics with catastrophe, rescue, and nucleation processes, Phys Rev E Stat Nonlin Soft Matter Phys 80 (2009), 031904.

[JG15] V Jemseena and M Gopalakrishnan, Effects of aging in catastrophe on the steady state and dynamics of a microtubule population, Phys Rev E Stat Nonlin Soft Matter Phys 91 (2015), no. 5.

[MFB+12] SP Maurer, FJ Fourniol, G Bohner, CA Moores, and T Surrey, Ebs recognize a nucleotidedependent structural cap at growing microtubule ends, Cell 149 (2012), no. 2, 371-82.

[MK14] N Müller and J Kierfeld, Effects of microtubule mechanics on hydrolysis and catastrophes, Phys Biol 11 (2014), no. 4, 046001. 